\title{
ON THE FITTING CORE OF A FORMATION
}

\author{
J.C. Beidleman and H. Heineken
}

\section{Dedicated to the memory of Bernhard H. Neumann}

\begin{abstract}
Not often is a formation also a Fitting class. In this article groups are exhibited that are contained in a given saturated formation and that do not lead out of this formation by forming normal products with other groups of it. This generalises a result of Cossey on $T$-groups and supersolvable groups.
\end{abstract}

\section{INTRODUCTION}

In this note we shall be concerned with an aspect of formations of solvable groups, especially of saturated formations. If a class of groups is a formation, it is closed with respect to forming quotient groups and subdirect products. Very quickly one is confronted with the limitation of this concept: in particular, the class of supersolvable groups - an example that comes to mind easily - is not closed with respect to normal products, one of the two criteria for being a Fitting class. The Fitting core $\mathcal{D}$ of a class $\mathcal{C}$ defines the subclass of groups that do not lead out of the class. In other words, the class $\mathcal{D}$ is defined by the rule

$\left(^{*}\right)$ If $A \in \mathcal{D}$ and $B \in \mathcal{C}$ and $A$ and $B$ are normal subgroups of $\langle A, B\rangle$, then $A B \in \mathcal{C}$.

Notice that this implies $\mathcal{D} \subseteq \mathcal{C}$ since the trivial group belongs to $\mathcal{C}$. Cossey (see [2]) has shown that the class of $T$-groups belongs to the Fitting core of the class of supersolvable groups. While quotient groups of $T$-groups are again $T$-groups we shall see that the Fitting core of a formation will in general not satisfy any of the usually considered closure properties. The main reason for this deficiency seems to be, that the automorphism group of a group and the automorphism groups of its subgroups and quotient groups are only loosely connected with each other.

In the case of saturated formations we are able to give a description via the automorphism groups induced by the group on the $p$ - chief factors (local description, see $[4$, p. 356] for particulars). If the local description consists of Fitting formations, then the formation described is also a Fitting formation, and we have the formation as its own Fitting core. In the other case we shall need a more complicated description for our groups.

Received 13th January, 2003

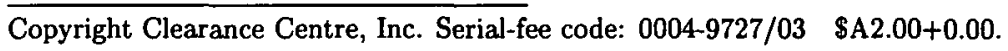




\section{Chief SERIES AND CharaCteristic SERIES}

We adopt the notation found, for instance, in Huppert [5, p. 64]: A chief series of a finite group $G$ is a densest series of normal subgroups of $G$ so that a chief factor $H / K$, the quotient of two consecutive terms, is a minimal normal subgroup of $G / K$. A characteristic series of a finite group $G$ is a densest series of characteristic subgroups, the quotient of two consecutive terms is called a characteristic factor.

It is surely well known that a saturated formation can be described via chief factors as well as via characteristic factors. The main step is given by the following statement.

LEMMA 1. The following two statements on a finite group $G$ are equivalent.

(i) If $H / K$ is a chief factor of $G$ and a p-group, then $(G / K) / C_{G / K}(H / K)$ belongs to the formation $\mathcal{F}$.

(ii) If $H / K$ is a characteristic factor of $G$ and a $p$-group, then $(G / K) / C_{G / K}$ $(H / K)$ belongs to the formation $\mathcal{F}$.

For a proof remember that a characteristic factor is a direct product of isomorphic copies of some chief factor. The quotient group of the centraliser in (i) is therefore an epimorphic image of some quotient group in (ii), in the other direction we have a subdirect product.

\section{Generalised POWER MAPPings}

In the sequel we shall consider groups with descriptions on how the group induces automorphism groups on characteristic factors where the actual representation as linear mapping shall be important. To that purpose we single out special linear mappings.

DEFINITION: Let $\sigma$ be a linear mapping of order a power of a prime $p$ on a vector space over a field of order a prime $q$. We call $\sigma$ a generalised power mapping, if the following is true: The minimal polynomial of $\sigma$ is irreducible and of degree prime to $p$. In general, the linear mapping $\sigma$ on a vector space over a field of order a prime $q$ is a generalised power mapping if all powers of $\sigma$ that are of prime power order are generalised power mappings in the sense outlined before.

REMARK. 1. A linear mapping of order a power of $q$ over a field of order $q$ is never irreducible. So the definition infers that the linear mapping has to be of order prime to $q$ whenever it is a generalised power mapping over a field of order $q$.

2. If a linear mapping over a vector space of characteristic $q$ is of square free order and possesses an irreducible minimal polynomial, then it is a generalised power mapping.

3. If the minimal polynomial of a linear mapping $\sigma$ of a vector space over a field of order $q$ is irreducible, then the power $\sigma^{k}$ has the same minimal polynomial as $\sigma$ if and only if $k \equiv q^{s}$ modulo the order of $\sigma$, for some integer $s$; compare L. E. Dickson [3, Theorems 24 and 25, p. 15 and 16]. This will be important for the possible automorphisms in our group constructions. 
4. Consider a linear mapping of order 21 of a vector space over the field of order 11. Irreducible minimal polynomials of such a mapping will have degree 6 , and $\operatorname{gcd}(6,21)$ $=3 \neq 1$. On the other hand, if the mapping has irreducible minimal polynomial it is a generalised power mapping by Remark 2 . This shows that the obvious "abbreviation" of the given definition leads to more restriction.

The following statement turns out to be crucial for this concept.

Lemma 2. Assume that the group $G$ is an extension of an elementary Abelian $q$ group $A$ by a cyclic group $\langle x\rangle$ of order prime to $q$ such that $A=C(A)$ and $x$ induces by conjugation a generalised power mapping on $A$. If $\tau$ is any automorphism of $G$, then $\left\langle x^{-1} \tau(x), A\right\rangle / A$ is a direct factor of $G / A$.

ProOF: The cyclic group $\langle x\rangle$ is the direct product of its subgroups of maximal prime power order. Let $\langle y\rangle$ be one of these subgroups of maximal prime power order, for instance of order $p^{s}$. By construction, $\tau(\langle y, A\rangle)=\left\langle y^{k}, A\right\rangle$ where $k$ is some power of $q$ (see Remark 3). The restriction of $\tau$ to $\langle y, A\rangle / A$ has order a divisor of the degree of the minimal polynomial of the linear mapping induced by $y$ in $A$ by conjugation, and this number is prime to $p$ by hypothesis. So either the restriction of $\tau$ is the identity or $\left\langle y^{-1} \tau(y), A\right\rangle=\langle y, A\rangle$. Proceding like this for all maximal prime power order subgroups leads to the statement of the Lemma.

We are now ready for our main result.

Local Theorem. Let $\mathcal{F}$ be a formation of solvable groups, and let $G$ be a finite normal product of the two solvable subgroups $A, B$ satisfying the following conditions:

(i) If $H / K$ is a $p$-chief factor of $A$, then

$$
(A / K) / C_{A / K}(H / K) \in \mathcal{F} .
$$

(ii) If $U / V$ is a $p$-group and a characteristic factor of $B$, then $(B / V) / C_{B / V}(U / V)$ is a cyclic group belonging to $\mathcal{F}$ and the automorphisms induced by $B / V$ in $U / V$ are generalised power automorphisms.

Then the normal product $G=A B$ satisfies condition (i), with $G$ taken for $A$.

Proof: We have to consider $p$-chief factors $R / S$ of $A B$. For this we choose a chief series containing $A \cap B$ and $A$. For $A \subseteq S$ the statement is true because of $A B / A \cong B / A \cap B$ and (i) is true by Lemma 1. For $A \cap B \subseteq S \neq R \subseteq A$ the statement is true by the hypothesis on $A$. Let now $R \subseteq A \cap B$. Then $R / S$ is a direct product of minimal $A$-invariant factors $R_{i} / S$ and so $(A / S) / C_{A / S}(R / S)$ is a subdirect product of the quotients $(A / S) / C_{A / S}\left(R_{i} / S\right)$ and so belongs to $\mathcal{F}$. On the other hand, $R / S$ is a quotient of a characteristic factor of $B$, and $(B / S) / C_{B / S}(R / S)$ is cyclic, belongs to $\mathcal{F}$ and the automorphisms induced are generalised power automorphisms. For brevity, we denote the centralisers $C_{A / S}(R / S), C_{B / S}(R / S), C_{A B / S}(R / S)$ simply by $C_{A}, C_{B}, C_{A B}$. Now

$$
(A B / S) / C_{A B}=\left((A / S) C_{A B} / C_{A B}\right)\left((B / S) C_{A B} / C_{A B}\right)
$$


and $(A / S) C_{A B} / C_{A B} \cong\left((A / S) / C_{A}\right)$ while $(B / S) C_{A B} / C_{A B} \cong(B / S) / C_{B}$. Now $(B / S) / C_{B}$ is cyclic and the induced operation is a generalised power mapping, and Lemma 2 shows that the normal subgroup $(B / S) C_{A B} / C_{A B}$ is a direct product

$$
(D / S) C_{A B} / C_{A B} \times(E / S) C_{A B} / C_{A B}
$$

where the first factor $(D / S) C_{A B} / C_{A B}$ is centralised by $(A / S) C_{A B} / C_{A B}$, while for the second we have

$$
\left[(E / S) C_{A B} / C_{A B},(A / S) C_{A B} / C_{A B}\right]=(E / S) C_{A B} / C_{A B} \subseteq(A / S) C_{A B} / C A B
$$

We obtain that in fact $A B=A D$ and $(A D / S) / C_{A B}$ is a central product of the factors $(A / S) C_{A B} / C_{A B}$ and $(D / S) C_{A B} / C_{A B}$, both belonging to $\mathcal{F}$. Since formations are closed with respect to epimorphic images and direct products, they are also closed with respect to central products, and (i) is true for the chief factor $R / S$ in every case. The Local Theorem is shown.

Via the local description of saturated formations, the local Theorem leads us to the following

Proposition. Let $\mathcal{F}$ be a saturated formation of solvable groups, described locally by formations $\mathbf{f}_{\mathrm{p}}$.

Let $T$ be a group satisfying the following conditions:

(i) $T \in \mathcal{F}$,

(ii) if $\mathrm{f}_{\mathrm{p}}$ is a non-empty non-Fitting formation and $H / K$ is a characteristic factor of $T$ and a $p$-group, then $(T / K) / C_{T / K}(H / K)$ is cyclic and elements of $T / K$ operate as generalised power automorphisms on $H / K$.

Then $T$ belongs to the Fitting core of $\mathcal{F}$.

Proof: A local "formation" $\mathbf{f}_{\mathbf{p}}$ being empty means that the groups contained in the formation $\mathcal{F}$ are of order prime to $p$; for this part condition (i) is sufficient, also for the case that $\mathbf{f}_{p}$ is a Fitting formation. We have to consider only the case of a non-empty non-Fitting formation, and here the result is a direct consequence of our Local Theorem.

Corollary 1 . Let $\mathcal{F}$ be a saturated formation of solvable groups, described locally by the formations $\mathbf{f}_{\mathbf{p}}$ and let $G$ be a group belonging to $\mathcal{F}$. Assume that if $\mathbf{f}_{\mathbf{p}}$ is a non-empty non-Fitting formation and $H / K$ is a characteristic p-factor of $G$, then $G$ induces by conjugation on $H / K$ power automorphisms. Then $G$ belongs to the Fitting core of $\mathcal{F}$.

A group $G$ is called a $P S T$-group if all the subnormal subgroups permute with all the Sylow subgroups of $G$. The class of PST-groups includes the class of $T$-groups. By a result in Agrawal [1] we know that the nilpotent residual of a solvable PST-group $G$ is an Abelian Hall subgroup of $G$ and all automorphisms induced by $G$ are power automorphisms. Therefore we obtain 
COROLlary 2. Let $G$ be a solvable PST-group belonging to a saturated formation $\mathcal{F}$. Then $G$ belongs to the Fitting core of $\mathcal{F}$.

Note that Corollary 2 includes Cossey's result mentioned earlier.

\section{EXAMPLES}

The Fitting core is a class of groups which will in general not be closed with respect to normal products and epimorphic images. This is shown by the first two examples.

1. Choose two odd primes $p, q$ such that $p$ divides $q-1$. Consider a normal extension $G$ of an elementary Abelian $q$-group $Q$ of rank $p+1$ by a nonabelian group $P$ of order $p^{3}$ and exponent $p$ such that $Z(G)=1$. The normal subgroup $Q$ splits into two normal subgroups of $G$, one ( $R$, say) of rank 1 and one $(S)$ of rank $p$. More explicitly, we assume that $P=\langle a, b\rangle$ operates on $S=\left\langle s_{1}, s_{2}, \ldots, s_{p}\right\rangle$ in the following

$$
\begin{gathered}
a^{-1} s_{i} a=s_{i}^{\alpha^{i}} \text { where } \alpha \not \equiv 1 \equiv \alpha^{p} \text { modulo } q, \\
b^{-1} s_{p} b=s_{1} ; b^{-1} s_{i} b=s_{i+1} \text { for } 1 \leqslant i<p,
\end{gathered}
$$

further assume $[a, R]=R$. Now $P$ is the product of the two normal elementary Abelian subgroups $A=\langle a,[a, b]\rangle$ and $B=\langle b,[a, b]\rangle$ of order $p^{2}$. By construction, $Z(A Q) \neq 1$. In turn, $A=\langle a\rangle \times\langle[a, b]\rangle$ and $\langle a, Q\rangle$ and $\langle[a, b], Q\rangle$ belong to the Fitting core of the formation of supersolvable $\{p, q\}$-groups: $[a, b]$ centralises $R$ and induces a non-identity power automorphism on $S$, while $Q$ splits into a direct product of factors, all except one of rank one, the remaining of rank two, such that $a$ induces different power automorphisms on these factors and does not centralise the factor of rank two. So $\langle[a, b], Q\rangle$ and $\langle a, Q\rangle$ both belong to the Fitting core of supersolvable $\{p, q\}$-groups.

We summarise: $G=P Q=(A Q)(B Q)$ is not supersolvable, $B Q$ is supersolvable, $A Q=\langle[a, b], Q\rangle\langle a, Q\rangle$ does not belong to the respective Fitting core while $\langle[a, b], Q\rangle$ and $\langle a, Q\rangle$ do. So there is no closure with respect to normal products.

Remark to EXAmple 1. For $p=2$ it suffices to choose $G=P Q$ such that $P$ is dihedral of order 8 and $Q$ is elementary Abelian of rank 2 .

2. Let $p, q$ as in the first example. Choose a number $a$ such that $1<a<q$ and $a^{p}-1$ is divisible by $q$. The following extension of an elementary Abelian $q$-group $Q=\langle x(i, j), y(k) \mid k \in\{0, \ldots, p-1\} ; 1 \leqslant j \leqslant i \leqslant 3\rangle$ of rank $p+6$ by an elementary Abelian $p$-group $P=\langle c, d\rangle$ of rank 2 defined by the relations below belongs to the Fitting core of the supersolvable $\{p, q\}$-groups: $c^{-1} z c=z^{a}$ for all $z \in Q, d^{-1} y(k) d=y(k)^{a^{k}}$ for $0 \leqslant k<p, d^{-1} x(i, j) d=x(i, j)^{a^{i}}$ for $1 \leqslant j \leqslant i \leqslant 3$.

This group $H=P Q$ possesses $p-3$ minimal characteristic subgroups of rank 1 namely $\langle y(k)\rangle$ for $k=0$ and $4 \leqslant k<p$, and one each of rank 2,3 and 4, namely $\langle y(1), x(1,1)\rangle,\langle y(2), x(2,1), x(2,2)\rangle$ and $\langle y(3), x(3,1), x(3,2), x(3,3)\rangle$. The centralisers of the latter three intersect with $P$ in the subgroups $\left\langle d c^{-1}\right\rangle,\left\langle d c^{-2}\right\rangle$ and $\left\langle d c^{-3}\right\rangle$. These 
three subgroups of $P \cong H / Q$ are fixed by all automorphisms which are induced by automorphisms of $H$. This yields that all subgroups of $H / Q$ are fixed, and automorphisms of $H$ induce a power automorphism on $H / Q$ (in fact, the identity automorphism). On the other hand, $\langle y(k)\rangle, c, d \mid 0 \leqslant k \leqslant p-1$ is isomorphic to a quotient group $H / R$ of $H$ and does not belong to the Fitting core of the formation of supersolvable $\{p, q\}$-groups. We have seen this in Example 1.

REMARK TO EXAMPLE 2. For $p=2$ the following construction leads to the analogue statement: $P=\langle c, d\rangle, Q=\left\langle x_{1}, x_{2}, x_{3}\right\rangle$, and the following relations hold:

$$
\begin{gathered}
c^{2}=d^{2}=[c, d]=1 \\
x_{i}^{q}=\left[x_{i}, x_{j}\right]=1 \text { for } 1 \leqslant i, j \leqslant 3 \\
\left(x_{1} c\right)^{2}=\left(x_{2} c\right)^{2}=\left(x_{3} d\right)^{2}=\left[x_{1}, d\right]=\left[x_{2}, d\right]=\left[x_{3}, c\right]=1 .
\end{gathered}
$$

The final example shows that the condition on the degree of the minimal polynomial in the definition of generalised power automorphisms is indispensible.

3. Let the prime $p$ be a divisor of $q^{k}-1$, where $q$ is a prime and $k$ is not divisible by $p$. Further let $k$ be minimal with this property and assume that $m$ is maximal such that $p^{m}$ divides $q^{k}-1$ (For $p=2$ assume $m>1$ ). There is an extension of an elementary Abelian $q$ - group $Q$ of rank $p k$ by a $q$-group of nilpotency class 2 , namely $P=\left\langle a, b \mid a^{p^{m+1}}=b^{p}=a^{-\left(1+p^{m}\right)} b^{-1} a b=1\right\rangle$, such that $Z(P Q)=1$. The group $P$ possesses two cyclic normal subgroups $\langle a\rangle,\langle a b\rangle$ of order $p^{m+1}$. Both $\langle a, Q\rangle$ and $\langle a b, Q\rangle$ belong to the saturated formation of extensions of nilpotent $\{p, q\}$-groups by Abelian groups of exponent $p^{m+1}$ and they do not belong to the corresponding Fitting core.

REMARK TO EXAMPLE 3. For $q=4 k-1$ choose for $Q$ an elementary Abelian group of rank 2 and for $P$ the quaternion group of order 8 .

\section{REFERENCES}

[1] R.K. Agrawal, 'Finite groups whose subnormal subgroups permute with all Sylow subgroups', Proc. Amer. Math. Soc. 47 (1975), 77-83.

[2] J. Cossey, 'Finite groups generated by subnormal T-groups', Glasgow Math. J. 37 (1995), 363-371.

[3] L.E. Dickson, Linear groups with an exposition of the Galois field theory (Dover Publications, New York, 1958).

[4] K. Doerk and T. Hawkes, Finite soluble groups, de Gruyter Expositions in Mathematics 4 (de Gruyter and Co., Berlin, 1992).

[5] B. Huppert, Endliche Gruppen I (Springer-Verlag, Berlin and New York, 1967).

Department of Mathematics

University of Kentucky

Lexington KY 40506-0017

United States of America

e-mail: clark@ms.uky.edu
Mathematisches Institut

Universität Würzburg

Am Hubland

97074 Würzburg

Germany

e-mail: Heineken@mathematik.uni-wurzburg.de 